\title{
ANCORAMENTO DOS CONTORNOS DE GRÃO EM UMA LIGA Al - I\%MASSA Mn: COMPARAÇÃO DE EXPERIMENTOS COM MODELOS ANALÍTICOS
}

\author{
Gláucio Soares da Fonseca \\ Paulo Rangel Rios ${ }^{2}$
}

\section{Resumo}

A expressão usual para calcular o raio limite do grão devido ao ancoramento de partícula é $R_{L}=A r / f$, onde $r$ é o raio da partícula, $f$ é a fração volumétrica, $R_{L}$ é o raio limite do grão e $A$ é uma constante igual a $1 / 6$ de acordo com Rios.(I) Essa formulação não é a mais conveniente para comparação com resultados experimentais. Neste trabalho são propostas duas expressões : 1) $S_{V L}=3 S_{V p}$, onde $S_{V}$ é a área do contorno de grão por unidade de volume e $S_{V p}$ é a área interfacial por unidade de volume e 2) $H=S_{v p}$, onde $H$ é a curvatura média do contorno de grão. Essas expressões são comparadas com medidas experimentais realizadas em uma liga de $\mathrm{Al}$ - 1 \%massa $\mathrm{Mn}$ de alta pureza contendo precipitados $\mathrm{Al}_{6} \mathrm{Mn}$, tratada termicamente por 3600 s em temperaturas variando de $500 \mathrm{a} 620^{\circ} \mathrm{C}$. Boa concordância é obtida entre teoria e os experimentos. Os méritos e defeitos de cada expressão são discutidos em detalhes de um ponto de vista fundamental e experimental. Conclui-se que a primeira expressão é a melhor escolha para propostas práticas. Palavras-chave: Crescimento de grão; Ancoramento de partícula; Curvaturas.

\section{GRAIN BOUNDARY PINNING IN An AI - IMASS\%Mn ALLOY: COMPARISON OF EXPERIMENTS WITH ANALYTICAL MODELS}

\begin{abstract}
The usual expression to calculate the limiting grain radius due to particle pinning is $R_{L}=A r / f$, where $r$ is the particle radius, $f$ is the volume fraction of the particles, $R_{L}$ is the limiting grain radius and $A$ is a constant equal to $I / 6$ according to Rios. (I) This form is not the most convenient for comparison with experimental measurements. In this work two alternative expressions are proposed: I) $S_{V L}=3 S_{V p}$, where $S_{V}$ is the grain boundary area per unit of volume and $S_{V P}$ is the interface area per unit of volume and 2) $H=S_{V p}$, where $H$ is the average grain boundary curvature. These expressions are compared with experimental measurements carried out in a high purity $\mathrm{Al}$ - Imass $\% \mathrm{Mn}$ alloy containing $\mathrm{Al}_{6} \mathrm{Mn}$ precipitates annealed for $3600 \mathrm{~s}$ at temperatures ranging from 500 to $620^{\circ} \mathrm{C}$. Good agreement is obtained between theory and experiment. The relative merits and shortcomings of each expression are discussed in details from a fundamental and experimental point of view. It is concluded that the first expression is probably the best choice for practical purposes.
\end{abstract}

Key words: Grain growth; Particle pinning; Grain boundary curvature.

\section{INTRODUÇÃO}

Muitas ligas comerciais contêm uma dispersão de partículas finas com a proposta específica de controlar o crescimento de grão. Ligas de alumínio e aços são exemplos bem conhecidos. Por exemplo, em uma siderúrgica uma grande quantidade de aços, na ordem de milhöes de toneladas, contém um pequeno teor de titânio. Titânio combinado com nitrogênio forma uma dispersão de $\mathrm{TiN}$, que controla o crescimento de grão durante o reaquecimento das placas.
Existem razỗes fundamentais como práticas para haver uma relação entre as características da dispersão de partículas e o raio crítico ou limite do grão.(1-6) Uma relação é provavelmente mais usada se ela contém quantidades que podem ser prontamente medidas por uma seção plana. Um problema peculiar a esse campo é que existe um grande número de modelos teóricos, mas um número pequeno de resultados experimentais. $(4-6)$

\footnotetext{
IEngenheiro Químico, Mestre em Engenharia Metalúrgica, Aluno do curso de Doutorado em Engenharia Metalúrgica na Universidade Federal Fluminense. kottel@terra.com.br, glaucio@metal.eeimvr.uff.br

2 Professor Ph.D, Coordenador do Curso de Pós - graduaçäo em Engenharia Metalúrgica da EEIMVR/ UFF. prrios@metal.eeimvr.uff.br
} 
A equação usual do resultado teórico é: $R_{L}=\frac{r}{6 f}$,

em que $R_{L}$ é o raio limite do grão, $r$ é o raio da partícula, $f$ é a fração volumétrica das partículas. $O$ coeficiente numérico depende de detalhes do modelo teórico, 1/6 mostrado acima foi obtido por Rios,(1) enquanto em livros didáticos usa-se a equação clássica de Zener(2) com coeficiente numérico igual a 4/3.

Uma expressâo na qual as quantidades são mais simples de medir é desejável. Em 1987, Rios propôs uma expressão baseada na área interfacial por unidade de volume: $(2,5)$

$$
S_{V L}=3 S_{V P} \text { ou } \lambda=\frac{2}{3 S_{V P}} \text {, }
$$

em que $S_{V L}$ é a área do contorno de grão por unidade de volume, quando a matriz encontrou o raio limite do grão, $S_{V p}$ é a área interfacial por unidade de volume entre as partículas e $\lambda$ é o comprimento médio das intersecções dos grãos.

A Equação 2 é mais fácil de verificar experimentalmente e foi derivada novamente por Rios e Fonseca em um recente trabalho.(5) Ela pode ser convertida na Equaçãol por meio das Equações 3 e 4 :

$$
S_{V}=\frac{1,5}{R},
$$

em que $S_{V}$ é a área do contorno de grão por unidade de volume para um tamanho médio de grão da matriz igual a $R$, assumindo que todos os grãos são esferas iguais de raio $R$, e

$$
S_{L P}=\frac{3 f}{r},
$$

em que $S_{V p}$ é a área interfacial por unidade de volume entre as partículas e a matriz, assumindo que todas as partículas são esferas iguais de raio $r$ e fração volumétrica $f$.

Uma outra expressão foi recentemente(6) derivada baseada na curvatura do contorno de grão ao invés da área do contorno, ou seja:

$$
H_{L}=S_{V P},
$$

Agora, em vez de raio limite do grão tem-se a curvatura limite do contorno de grão, $H_{L}$. O crescimento de grão ocorrerá se a curvatura do contorno de grão da matriz, $H$, é maior que $H_{L}$. Se a curvatura do contorno de grão da matriz, $H$, é menor que $H_{L}$, a matriz é ancorada e crescimento de grão não ocorre.

É importante esclarecer a definição de $H$ adotada neste trabalho, em que é seguida a hipótese sugerida por Taylor.(7) Taylor sugeriu definir a curvatura local como a soma das duas principais curvaturas: $k_{1}$ e $k_{2}$. Essa definição difere da definição de $\operatorname{DeHoff}^{(8)}$ da integral da curvatura média, que inclui o fator $1 / 2$. A sugestão de Taylor é razoável porque a velocidade do contorno de grão é proporcional a $\mathrm{k}_{1}+\mathrm{k}_{2}$. $\mathrm{H}$ é denominado abreviadamente de curvatura do contorno de grão.

A Equação 5 pode ser transformada na Equação 2 por meio da relação entre a área do contorno de grão e a curvatura do contorno de grão durante o crescimento de grão, ou seja: $(3,5,6)$

$$
H=\frac{S_{V}}{3}=\frac{2}{3} \lambda^{-1}=\frac{0,5}{R},
$$

em que $\lambda$ é o comprimento médio das intersecções dos grãos.

Neste trabalho, as Equações 2 e 5 são testadas com os dados medidos em uma liga $\mathrm{Al}$ - I\%massaMn contendo precipitados $\mathrm{Al}_{6} \mathrm{Mn}$. A liga $\mathrm{Al}-\mathrm{I} \%$ massaMn é particularmente conveniente para este estudo porque a microscopia óptica pode ser usada para caracterizar a microestrutura.

\section{MATERIAIS E MÉTODOS}

Foi usada uma liga $\mathrm{Al}-\mathrm{I} \%$ massaMn de alta pureza, contendo um total de impurezas menor que $40 \mathrm{ppm}$. Detalhes do processamento e das técnicas experimentais podem ser encontradas em Rios e Fonseca. (5) Dois grupos de amostras foram estudados e laminados a frio: um com $40 \%$ e outro com $80 \%$. Depois da laminação a frio todas as amostras foram recristalizadas a $500^{\circ} \mathrm{C}$ por I hora e a seguir submetidas ao recozimento para o crescimento de grão. A temperatura de recozimento variou de $500^{\circ} \mathrm{C} \mathrm{a} 620^{\circ} \mathrm{C}$ em passos de $20^{\circ} \mathrm{C}$. A área do contorno de grão por unidade de volume, $S_{V}$, fração volumétrica e área interfacial por unidade de volume dos precipitados foram medidos por técnicas convencionais. (9)A integral da curvatura do contorno de grão foi medida usando o método da tangente de DeHoff. $(8,9)$ O número de pontos da tangente entre a linha teste e o contorno de grão curvado em uma seção por unidade de área teste, $T_{A}$, foi medido. Com $T_{A}$ e $S_{V}$ a curvatura do contorno de grão foi obtida empregando-se a expressão:

$$
H=\frac{2 \pi T_{A}}{S_{V}}
$$

Vale repetir que essa expressão difere por um fator de dois da original de $\operatorname{DeHoff}^{(8)}$, porque $H$ é definido aqui como sugerido por Taylor,(7) a soma das duas principais curvaturas $k_{1}$ e $k_{2}$, ao invés da média de $k_{1}$ e $k_{2}$, como estabelecido por DeHoff.(8)

\section{RESULTADOS}

As amostras deformadas $40 \%$ e $80 \%$ para recristalização serão referidas como amostra " $40 \%$ " ou " $80 \%$ ", respectivamente.

A fração volumétrica (vol\%) e a área interfacial por unidade de volume $\left(S_{V P}\right)$ de uma dispersão de partículas $\mathrm{Al}_{6} \mathrm{Mn}$ são apresentadas nas Figuras I e 2. A Figura I evidencia que os resultados das fraçôes volumétricas das amostras $40 \%$ são similares aos das amostras $80 \%$. Nesta figura são também apresentados valores do equilíbrio calculados com o software Thermo - Calc, versão TCW. Banco de dados: PBIN ( TC Public Binary Alloys Database (vl.l, 1997/2003) .A fração molar fornecida pelo software foi transformada em fração volumétrica através das densidades do alumínio e do $\mathrm{Al}_{6} \mathrm{Mn}$. 
Observa-se na Figura 2 que a área interfacial dos precipitados por unidade de volume são similares nas amostras $40 \%$ e $80 \%$. Portanto, a deformação prévia não teve influência na medida de dispersão de partícula. Com isso, o valor da área interfacial dos precipitados por unidade de volume foi obtido combinando os resultados de ambas as amostras.

A área do contorno de grão por unidade de volume, $S_{V}$, em função da temperatura de recozimento, é apresentada na Figura 3 para as amostras $40 \%$ e $80 \%$. As amostras $80 \%$ têm um tamanho de grão recristalizado menor que as amostras $40 \%$, significando que as amostras $80 \%$ têm uma área do contorno de grão por unidade de volume maior que as amostras $40 \%$. Nas amostras $80 \%$, para temperaturas de recozimento menores, abaixo de $540^{\circ} \mathrm{C}$, a área do contorno de grão permanece aproximadamente constante. A razão para isso é que o crescimento de grão somente ocorre quando a área limite do contorno de grão, representada por triângulos negros na Figura 3, é menor que a área do contorno de grão medida. De $500^{\circ}$ a $540^{\circ} \mathrm{C}$, a área do contorno de grão medida é menor que a área limite do contorno de grão e o crescimento de grão, que é, um decréscimo na área do contorno de grão, não pode ocorrer. Acima de $540^{\circ} \mathrm{C}$, as partículas dissolvem e a área limite do contorno de grão decresce com o aumento da temperatura de recozimento e a área do contorno de grão decresce seguindo a área limite do contorno de grão. As amostras $40 \%$ têm um comportamento similar, mas o crescimento de grão começa aproximadamente acima de $580^{\circ} \mathrm{C}$.

Refez-se a Figura 3 com o comprimento médio das intersecçōes dos grãos, $\lambda$, no lugar da área do contorno de grão por unidade de volume, $S_{V}$, usando para o cálculo do valor teórico a outra fórmula equivalente da Equação 2, como está apresentado na Figura 4.

A Figura 5 apresenta o gráfico da curvatura do contorno de grão em função da temperatura de recozimento. O comportamento é similar ao da área do contorno de grão.

\section{DISCUSSÃO}

Podem-se comparar os resultados das Figuras 3, 4 e 5 com os modelos obtidos na forma da Equaçãol, convertendo esses modelos com a ajuda das Equaçôes 3, 4 e 6. Assim, a equação clássica de Zener usada na literatura pode ser colocada na forma das Equaçốes 8 e 9

$$
\begin{aligned}
& S_{V L}=\frac{3}{8} S_{V P} \text { ou } \lambda=\frac{16}{3 S_{V P}}, \\
& H_{L}=\frac{1}{8} S_{V P} .
\end{aligned}
$$

Essas expressốes dão valores de $S_{V L}$ e $H_{L}$ bem abaixo dos obtidos experimentalmente e não foram colocadas nas Figuras 3, 4 e 5. Uma versão melhor do modelo de Zener, Zener " corrigido", $(3,5,6)$ é dada pelas Equações 10 e II:

$$
\begin{aligned}
& S_{V L}=\frac{3}{2} S_{V P} \text { ou } \lambda=\frac{4}{3 S_{V P}}, \\
& H_{L}=\frac{1}{2} S_{V P} .
\end{aligned}
$$

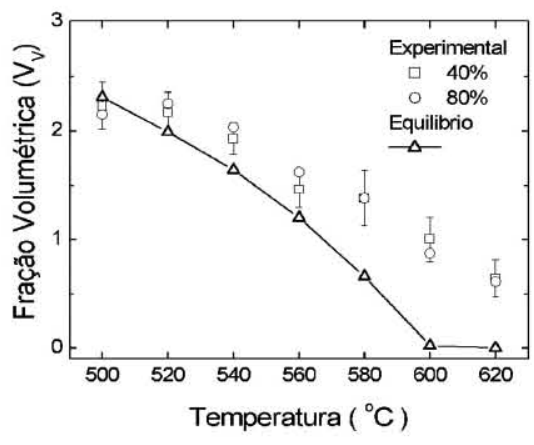

Figura I, $V_{v}$ em função da temperatura. Para facilitar a interpretaçäo, somente estäo mostradas as barras de erros nas amostras $40 \%$, já que os erros nas amostras $80 \%$ säo similares.

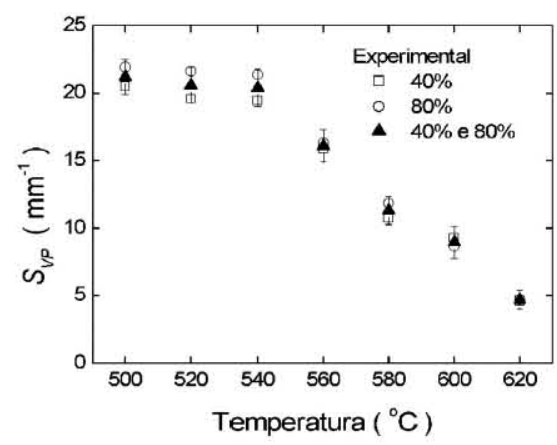

Figura 2. $S_{V P}$ em funçäo da temperatura. Os triângulos negros representam os valores obtidos pela combinação das medidas das amostras $40 \%$ e $80 \%$. Para facilitar a interpretaçäo, somente são apresentadas as barras de erros dos resultados combinados.

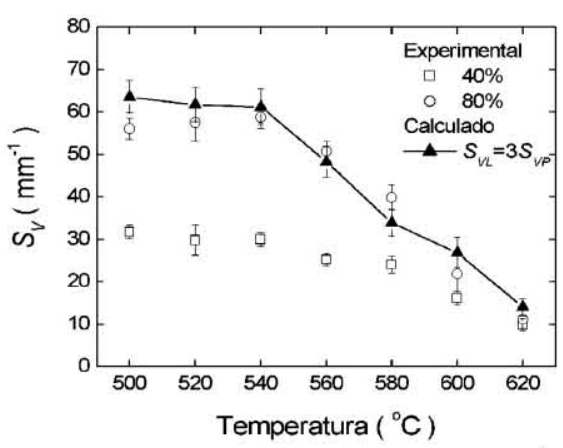

Figura 3. $S_{V}$, em função da temperatura. É também apresentado o valor teórico calculado, usando a Equação 2. A concordância entre a teoria e os experimentos é muito boa.

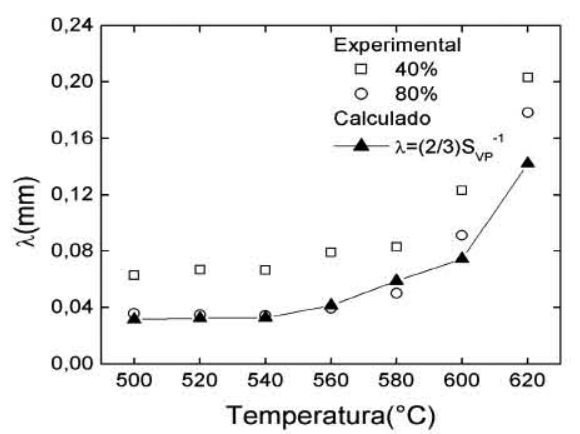

Figura 4. Comprimento médio das intersecçöes dos gräos, $\lambda$, em função da temperatura. É também apresentado o valor teórico calculado usando a Equaçäo 2. 


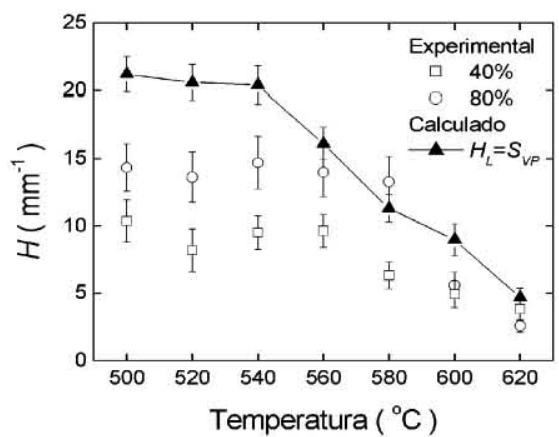

Figura 5. $H$ em funçäo da temperatura. É também apresentado o valor teórico calculado usando a Equação 5. A concordância entre a teoria e os experimentos é boa.

Os resultados para essas equações são melhores, mas ainda predizem áreas e curvaturas limites dos contornos de grãos abaixo do valor experimental nas temperaturas menores: $500^{\circ}$ $540^{\circ} \mathrm{C}$, ou seja, predizendo erroneamente que a matriz não é ancorada nessas temperaturas.

Os triângulos negros ligados por uma linha sólida nas Figuras 3, 4 e 5 representarn as Equações 2 e 5 . As Equações 2 e 5 são equivalentes, contudo as medidas experimentais requeridas para cada expressão são diferentes: somente $S_{V}$ (ou $\lambda$ ) para a Equação 2 e ambos $S_{V}$ e $T_{A}$ para a Equação 5. Para um ponto de vista fundamental, a curvatura do contorno de grão é mais satisfatória, já que a força motriz é medida diretamente. Por um outro lado, para um ponto de vista prático, $T_{A}$ requer um esforço extra, pois não é uma medida comum. A curvatura do contorno de grão envolve a razão de duas quantidades experimentais que estão sujeitas a erros. Esses erros podem ser a razão porque a concordância obtida na Figura 3, usando a Equação 2, e os dados experimentais correspondentes a ela é melhor que a concordância obtida na Figura 5 entre a Equação 5 e seus dados experimentais.

Portanto, na prática a Equação 2 é a melhor escolha.

\section{CONCLUSÃO}

A Equação 2 é a melhor escolha prática para descrever a relação entre a dispersão de partícula e a área do contorno de grão. A razão principal para isso é que as quantidades envolvidas são comparavelmente mais fáceis de medir e têm menores erros que as requeridas pelas Equações I e 5 . Por conveniência a Equação 2 é repetida aqui:

$$
S_{V L}=3 S_{V P} \text { ou } \lambda=\frac{2}{3 S_{V P}},
$$

\section{Agradecimentos} FAPERI.

\section{REFERÊNCIAS}

I RIOS, P.R. A theory for grain boundary pinning by particles. Acta Metallica Materialia, v. 35, n. 12, p.2805-28|4, Dec. 1987.

2 ZENER, C. Comunicação privada apud SMITH, C.S. Grains, phases, and interfaces: an interpretation of microstructure. Transactions of The American Institute of Mining and Metallurgical Engineers, v. I75, p. 47, 1948.

3 RIOS, P.R. On the relationship between pinning force limiting grain radius. Scripta Materialia., v. 34, n.8, p. I I85I I88, Abr. 1996.

4 MANOHAR, P.A.; Ferry, M.; Chandra, T. Five decades of the Zener equation. ISIJ International, v. 38, n.9, p. 9I3924, 1998.

5 RIOS, P.R.; FONSECA, G.S. Grain boundary pinning by $\mathrm{Al}_{6} \mathrm{Mn}$ precipitates in an $\mathrm{Al}-\mathrm{Iwt} \% \mathrm{Mn}$ alloy. Scripta Materialia., v. 50, n.I, p. 7I-75, Jan. 2004.

6 RIOS, P.R.; FONSECA, G.S. Grain boundary curvature and particle pinning in an Al-Imass\% Mn alloy. Scripta Materialia., v.50, n. II, p. 1373-1377, Jun. 2004.

7 TAYLOR, J.E. II - Mean curvature and weighted mean curvature. Acta Metallica Materialia, v. 40, n.7, p. I4751485, Jul. 1992.

8 DEHOFF, R.T. The quantitative estimation of mean surface curvature. Transactions of the Metallurgical Society of The American Institute of Mining and Metallurgical Engineers, v. 239, n.5, p. 617-62I, May 1967.

9 RUSS, J.C.; DEHOFF, R.T. Practical stereology. 2.ed. New York: Kluwer Academic, 2000.

I0 PATTERSON, B.R.; LIU, Y. Relationship between grain boundary curvature and grain size. Metallurgical Transactions, v. 23A, n.9, p. 248I-2482, Sept. 1992.

Recebido em: 15/02/2005

Aceito em: 07/I I/2005

Proveniente de: CONGRESSO ANUAL DA ABM - INTERNACIONAL, 59., 2004, São Paulo. São Paulo: ABM, 2004. 